\title{
Rapidly Progressive IgA Nephropathy: A Case Report With Review of Clinical Presentation, Prognostic Factors and Therapeutic Modalities
}

\author{
Isaac Akkad ${ }^{\mathrm{a}, \mathrm{b}}$, Alberto Ortiz ${ }^{\mathrm{a}}$, Melvyn Hecht ${ }^{\mathrm{a}}$
}

\begin{abstract}
IgA nephropathy (IgAN) is the most common glomerulonephritis in the Western world. Generally, the diagnosis of IgAN is based on clinical history, laboratory data, and histopathological reports. The rapidly progressing and crescentic subtype is quite uncommon. The clinical picture and spectrum of severity upon presentation varies between individual cases. In this case report, we present a previously healthy 20-year-old Latin American male with anti-neutrophil cytoplasmic antibodies seropositive rapidly progressing IgAN leading to severe acute renal impairment, uremic pericarditis, and uremic encephalopathy. Prognostic markers and histopathological types of injury have been proposed as tools to assess severity of disease and in guiding therapeutic options. This case report highlights histopathological, serological, and clinical characteristics of severe IgAN.
\end{abstract}

Keywords: Anti-neutrophil cytoplasmic antibodies; Rapidly progressive glomerulonephritis; Oxford classification; Absolute renal risk score

\section{Introduction}

An abnormal glycosylated immunoglobulin A with deficient galactose residues can induce autoantibody synthesis and form circulating immune complexes that can deposit in the renal mesangium [1]. The antigen-antibody complexes in the renal mesangium can ignite an inflammatory cascade with subsequent complement system activation and infiltration by lymphocytes, granulocytes, monocytes, and macrophages [2]. Patients with IgA nephropathy (IgAN) typically present with a single or recurrent episodes of visible hematuria, usually following an upper respiratory infection, or with mild proteinuria

\footnotetext{
Manuscript accepted for publication May 03, 2016

aDepartment of Medicine, Maimonides Medical Center, Brooklyn, New York 11219, USA

${ }^{b}$ Corresponding Author: Isaac Akkad, Department of Medicine, Maimonides Medical Center, Brooklyn, New York 11219, USA.

Email: iakkad@maimonidesmed.org
}

doi: http://dx.doi.org/10.14740/jmc2498w and microscopic hematuria detected during routine examination [3]. A small percentage of IgAN patients present with nephrotic syndrome or acute rapidly progressive glomerulonephritis $[4,5]$. The Oxford classification, absolute renal risk score, and other novel prognostic tools can aid in the classification of IgAN and help prognosticating therapeutic response and progression to end-stage renal disease (ESRD) [6-8].

\section{Case Report}

A 20-year-old Latin American male with no past medical history presented to the emergency room (ER) with 2 weeks of fatigue, lethargy, shortness of breath, mid sternal exertional chest pain worsened on inspiration, bilateral lower extremity swelling, nausea, and reporting brown emesis 4 - 5 times/day. He made urine and noted no color changes or dysuria. He denied recent travel. Of note, he had a sore throat 3 weeks ago for which he took acetaminophen only. Upon presentation, his vitals were significant for hypertension 164/109. Labs were significant for normocytic anemia 8.2/25.3, metabolic acidosis $\mathrm{pH} 7.22, \mathrm{HCO}_{3} 16, \mathrm{PCO}_{2} 40$, anion gap 18 , and $\mathrm{BUN} / \mathrm{Cr}$ 182/16.2. U/A showed $300 \mathrm{mg} / \mathrm{dL}$ of protein and large hemoglobin but no gross hematuria. Troponin I was $<0.03$, BNP was 732, and HIV was negative. Utox was negative, and radiographic examination of the chest was normal. EKG showed T-wave inversions in leads I, V5, V6 and prolonged QTc 505. The MICU and nephrology teams were immediately called and recommendations appreciated. PTH $451 \mathrm{pg} / \mathrm{mL}$, vitamin D 1,25 level of 9.0, normal complement, positive anti-neutrophil cytoplasmic antibodies (ANCA), negative ASO titters, elevated protein/Cr ratio, and elevated urine random creatinineprotein were shown. Echocardiogram and kidney ultrasound were performed. Placement of a dialysis catheter took place for emergent dialysis. We attributed the lethargy and headache to uremic encephalopathy, the SOB and chest pain to uremic pericarditis (echocardiogram findings: left ventricular ejection fraction, 45-50\%; mild to moderate aortic, tricuspid, pulmonic regurgitation and moderate to severe mitral valve regurgitation; moderately elevated pulmonary artery systolic pressure and present pericardial effusion). The generalized edema could be explained by the kidney failure. Initially in the differential post-streptococcal glomerulonephritis and renal artery stenosis were ruled out. Given his apparent rapid deterioration in kid- 
ney function, we hypothesized that a rapidly progressing nephropathy could be responsible and a kidney biopsy was performed. While waiting for the final pathological report, renal replacement therapy (RRT), blood pressure control, and immunosuppression were instituted. On light microscopy, there were approximately 7 - 12 glomeruli, in which 3 - 6 glomeruli (43-50\%) were globally sclerosed. Additional three glomeruli showed segmental scaring/sclerosis. Approximately 1 - 3 glomeruli (14-25\%) showed cellular to fibrocellular crescents. One glomerulus showed segmental necrosis. Overt intact glomeruli were not seen. The relatively intact areas showed marked mesangial expansion by matrix. There was moderate to severe interstitial fibrosis and tubular atrophy, associated with mild interstitial inflammation. Clusters of eosinophils were also seen in corticomedullary junction areas. Arteries were without significant histological abnormalities. Arteritis was not seen.

On direct immunofluorescence examination of the renal cortex, containing 4 - 6 glomeruli per level section, one glomerulus was globally sclerosed. There was granular staining in mesangial areas for IgA (2-3+), C3 (2+), kappa (1+), and lambda $(3+)$ light chains.

Electron microscopy showed immune type electron dense deposits in mesangial areas. Overt electron dense deposits were not seen in subepithelial or subendothelial locations. The glomerular capillary basement membranes were within a normal ultrastructure architecturally. The foot processes of podocytes were moderately effaced. Mesangial areas showed mild to moderate increased matrix, admixed with deposits.

Patient was diagnosed with IgAN with cellular to fibrocellular crescents (14-25\%), global glomerulosclerosis (43-50\%), severe interstitial fibrosis, and tubular atrophy associated with interstitial inflammation. Oxford classification was Mx Ex S1 $\mathrm{T} 2$.

The patient was not a candidate for kidney transplant at this time given his legal and insurance status. His progression to ESRD warranted an AV fistula creation for further dialysis needs. Pulse steroids given for 3 days followed by taper course, immunomodulation therapy, and RRT were his therapeutic options.

\section{Discussion}

Despite IgAN is the most common glomerulonephritis in the Western world, a particular rapid progression to ESRD is uncommon $(<10 \%)$ [9]. In this patient, the rapid clinical deterioration and histopathological presence of cellular/fibrous crescents, fibrinoid necrosis, and global sclerosis were suggestive of active glomerular injury in a background of cortical chronic injury.

In a study by Huang et al, 14 patients with IgAN and serological positive ANCA were reviewed to describe the clinical and histological characteristics of this patient subpopulation and assess if ANCA seropositive patients have higher odds of developing rapidly deteriorating kidney function [10]. In summary, they believed that patients with $\operatorname{IgAN}$ and pathogenic ANCAs commonly show clinical features of rapidly deterio- rating kidney disease [10]. In a different study, Yang et al [11] described the clinical and histological features, the response to treatment and the renal outcomes of 20 IgAN patients with positive serology for ANCA. Of 1,729 IgAN patients studied between 1997 and 2013 in which ANCA serology was available, 1.2\% (20 patients) were ANCA+ [12].

In a study from Lv et al [13], 113 Chinese patients with crescentic IgAN were recruited from eight kidney centers across China. It was found that patients with crescentic IgAN had a poor prognosis, and nearly $70 \%$ of these patients progressed to ESRD in 5 years, including those patients who had received immunosuppressive therapy. The initial serum creatinine $(\mathrm{SCr})$ concentration was the strongest risk factor for kidney failure. Using logistic curves, they developed a simple model based on SCr concentration to predict the outcomes of crescentic IgAN. In this model, a plot of the association between the probability of ESRD and the initial SCr formed an S-shaped curve. Patients with early stage disease $(\mathrm{SCr}<2.7$ $\mathrm{mg} / \mathrm{dL}$, the first turning point) had a good prognosis, with less than $25 \%$ of patients progressing to ESRD during follow-up. In contrast, patients with $\mathrm{SCr}>6.8 \mathrm{mg} / \mathrm{dL}(600 \mu \mathrm{mol} / \mathrm{L}$, the second turning point) were less likely to recover from chronic dialysis, even after aggressive immunosuppressive therapy [13].

Our patient presented with $\mathrm{SCr}>16 \mathrm{mg} / \mathrm{dL}, \mathrm{BUN}$ of 182 , along with rapidly deteriorating clinical course and positive serology for ANCA. It was hypothesized with the multi-disciplinary team that despite pulse steroids and immunomodulation, our patient was going to need long-term dialysis. He was kept with the temporary dialysis port for initial dialysis sessions. An arteriovenous fistula creation was performed for future needs. It was concluded that despite aggressive immunosuppressive therapy, the patient irreversibly was on ESRD. Due to the patient's legal and insurance status, we were unable to proceed and enlist the patient for kidney transplant at that time.

This case illustrates one of a small percentage of patients with $\operatorname{IgAN}$ that can develop a rapidly progressive form of the disease leading to ESRD.

Some clinical and laboratory findings at the time of diagnosis can potentially help stratify the severity of disease [14, 15]: elevated serum creatinine concentration with reduced GFR, hypertension ( $>140 / 90 \mathrm{~mm} \mathrm{Hg}$ ), or persistent proteinuria usually follows a worse prognosis. The magnitude of the effect of reduced GFR on prognosis was illustrated in a study from Japan of 2,270 patients with IgAN in which the cumulative incidence of ESRD at 7 years varied directly and markedly with the $\mathrm{SCr}$ at diagnosis ( $\mathrm{P}$ value for the trend $<0.001$ ) [11].

Although clinical features appear to serve as strong prognostic indicators, certain findings on renal biopsy in patients with IgAN have been associated with an increased risk of progressive disease. The Oxford histologic classification system may improve the ability to identify patients with a poor renal prognosis at the time of renal biopsy [16].

A combined prediction score that estimates the 5-year risk of developing ESRD known as the absolute renal risk score was developed in a Japanese cohort of 698 untreated patients and then validated in a separate cohort of 702 patients [17].

Patients were assigned points according to two clinical 
risk factors (24-h urine protein excretion and eGFR) and three of the four Oxford classification criteria (mesangial hypercellularity, segmental glomerulosclerosis, and tubular atrophy/ interstitial fibrosis).

In addition, a study of 97 patients with $\operatorname{IgAN}$ of varying severity found that higher titers of autoantibodies specific for poorly galactosylated IgA1 corresponded to both the absolute renal risk score (mentioned above) and the risk of ESRD or death [18]. Thus, testing for autoantibodies against abnormal IgA1 molecules may prove to be a useful prognostic tool in patients with $\operatorname{IgAN}$.

Other notable serologic markers that have been shown to correlate with severity of disease are ANCA as evaluated by Huang et al [10].

The optimal approach to the treatment of IgAN is still uncertain, with angiotensin-converting enzyme (ACE) inhibitors or angiotensin II receptor blockers (ARB) for blood pressure control and proteinuria.

The indications for the use of glucocorticoids alone or in combination with other immunosuppressive drugs in patients with $\operatorname{IgAN}$ are uncertain and not well defined.

In particular, the treatment of crescentic, rapidly progressive glomerulonephritis in patients with IgAN has not been evaluated in randomized trials. Observational data suggest possible benefit from regimens similar to those used in idiopathic crescentic glomerulonephritis consisting of intravenous pulse methylprednisolone followed by oral prednisone, intravenous or oral cyclophosphamide, eculizumab and/or plasmapheresis [19-21]. In this case report, we present an overview of rapidly progressing IgAN as seen in our patient. We used the available data to guide our clinical decisions and found ourselves with multiple studies with notable differences in amounts of patients, patient population, length to follow-up, selection of risk factors, and highly variable endpoints. We share the challenges of diagnosing, stratifying, and treating this variant of IgAN. We believe there is a need for unified diagnostic and prognostic criteria as well as a treatment option. By intervening early in the course of the disease, there might be an improvement in the clinical outcomes for this patient population.

\section{Conflicts of Interest}

The authors declare that there is no conflict of interest regarding the writing of this manuscript.

\section{References}

1. Mestecky J, Tomana M, Moldoveanu Z, Julian BA, Suzuki H, Matousovic K, Renfrow MB, et al. Role of aberrant glycosylation of IgA1 molecules in the pathogenesis of IgA nephropathy. Kidney Blood Press Res. 2008;31(1):29-37.

2. Barratt J, Smith AC, Molyneux K, Feehally J. Immunopathogenesis of IgAN. Semin Immunopathol. 2007;29(4):427-443.

3. Donadio JV, Grande JP. IgA nephropathy. N Engl J Med. 2002;347(10):738-748.
4. Rocatello D, Ferro M, Coppo R, et al. Treatment of rapidly progressive IgA nephropathy. In: Clarkson A, Woodroffe A (eds). IgA Nephropathy: Pathogenesis and Treatment. Basel: Karger, 1995, vol. 111, pp. 177-1483.

5. Habib R, Niaudet P, Levy M. Schonlein-Henoch purpura nephritis and IgA nephropathy. In: Tisher $\mathrm{C}$, Brenner BM (eds). Renal Pathology: with Clinical and Pathological Correlations, 2nd edn. Philadelphia, PA: Lippincott, 1994, pp. 472-523.

6. D'Amico G. Natural history of idiopathic IgA nephropathy and factors predictive of disease outcome. Semin Nephrol. 2004;24(3):179-196.

7. Donadio JV, Bergstralh EJ, Grande JP, Rademcher DM. Proteinuria patterns and their association with subsequent end-stage renal disease in IgA nephropathy. Nephrol Dial Transplant. 2002;17(7):1197-1203.

8. Wyatt RJ, Julian BA. IgA nephropathy. N Engl J Med. 2013;368(25):2402-2414.

9. Habib R, Jennette JC. Immunoglobulin A nephropathy and Henoch-Schonlein purpura. In: Fogo AB, Bruijn JA, Cohen AH, Colvin RB, Jennette JC (eds). Fundamentals of Renal Pathology. New York: Springer, 2006, pp. 61-69.

10. Huang X, Wang Y, Xie L, Zhang Y, Tang S, Yin S, Gao X, et al. IgA nephropathy with anti-neutrophil cytoplasmic antibody seropositivity. Clin Nephrol. 2015;84(3):156164.

11. Yang YZ, Shi SF, Chen YQ, Chen M, Yang YH, Xie XF, Zou R, et al. Clinical features of IgA nephropathy with serum ANCA positivity: a retrospective case-control study. Clin Kidney J. 2015;8(5):482-488.

12. Rojas-Rivera J, Fernandez-Juarez G, Praga M. Rapidly progressive IgA nephropathy: a form of vasculitis or a complement-mediated disease? Clin Kidney J. 2015;8(5):477-481.

13. Lv J, Yang Y, Zhang H, Chen W, Pan X, Guo Z, Wang C, et al. Prediction of outcomes in crescentic IgA nephropathy in a multicenter cohort study. J Am Soc Nephrol. 2013;24(12):2118-2125.

14. Wakai K, Kawamura T, Endoh M, Kojima M, Tomino Y, Tamakoshi A, Ohno Y, et al. A scoring system to predict renal outcome in IgA nephropathy: from a nationwide prospective study. Nephrol Dial Transplant. 2006;21(10):2800-2808.

15. Berthoux F, Mohey H, Laurent B, Mariat C, Afiani A, Thibaudin L. Predicting the risk for dialysis or death in IgA nephropathy. J Am Soc Nephrol. 2011;22(4):752761.

16. Coppo R, Troyanov S, Camilla R, Hogg RJ, Cattran DC, Cook HT, Feehally J, et al. The Oxford IgA nephropathy clinicopathological classification is valid for children as well as adults. Kidney Int. 2010;77(10):921-927.

17. Tanaka S, Ninomiya T, Katafuchi R, Masutani K, Tsuchimoto A, Noguchi H, Hirakata H, et al. Development and validation of a prediction rule using the Oxford classification in IgA nephropathy. Clin J Am Soc Nephrol. 2013;8(12):2082-2090.

18. Berthoux F, Suzuki H, Thibaudin L, Yanagawa H, Maillard N, Mariat C, Tomino Y, et al. Autoantibodies targeting galactose-deficient IgA1 associate with progression 
of IgA nephropathy. J Am Soc Nephrol. 2012;23(9):15791587.

19. Welch TR, McAdams AJ, Berry A. Rapidly progressive IgA nephropathy. Am J Dis Child. 1988;142(7):789-793.

20. McIntyre CW, Fluck RJ, Lambie SH. Steroid and cyclophosphamide therapy for IgA nephropathy associated with crescenteric change: an effective treatment. Clin Nephrol. 2001;56(3):193-198.

21. Tumlin JA, Lohavichan V, Hennigar R. Crescentic, proliferative IgAnephropathy: clinical and histological response to methylprednisolone and intravenous cyclophosphamide. Nephrol Dial Transplant. 2003;18(7):1321-1329. 